\title{
REFLEXIÓN SOBRE LA SITUACIÓN POLÍTICA, TERRITORIAL Y SOCIAL DEL PERÚ DESPUÉS DE LA INDEPENDENCIA
}

Pedro P. Soto Canales
Universidad Católica Sedes Sapientiae
psoto@ucss.edu.pe

Fecha de recepción: agosto de 2019 Fecha de aceptación: diciembre de 2019

Resumen: En este trabajo se abordará mediante un juicio crítico el proceso que acarreó nuestro tránsito de sociedad colonial a sociedad republicana. Para ello, se dará un repaso sistemático de documentos esenciales que influyeron en dicho proceso, al igual que el paralelo de los acontecimientos históricos dados a nivel internacional. Este análisis también tomará como base la historia de las ideas políticas, las cuales influyeron en el pensamiento

* Pedro P. Soto Canales es bachiller (1994) y licenciado (1996) en Educación en las especialidades de Ciencias Religiosas y Ciencias Histórico Sociales, por la Universidad Marcelino Champagnat. Es, también, diplomado en Docencia Universitaria (2004) por la Universidad Enrique Guzmán y Valle; diplomado en Seguridad y Defensa Nacional (2005) por el Ministerio de Defensa y la Universidad Católica Sedes Sapientiae y Magíster en Historia (2017) por la Universidad Nacional Mayor de San Marcos. Actualmente se encuentra realizando estudios de doctorado en Humanidades, con Mención en Estudios sobre Cultura, en la Universidad de Piura. Ejerce la docencia desde el año 1992 en los diversos niveles educativos. Actualmente es coordinador del área de Historia y de la carrera profesional de Turismo y Patrimonio Cultural en la Universidad Católica Sedes Sapientiae y docente de Historia de la Iglesia en la Facultad de Teología Redemptoris Mater (La Punta, Callao). 
de los personajes que ayudaron a construir la política y sociedad que abandonaba el modelo colonial.

Palabas clave: Historia del Perú, independencia del Perú, virreinato peruano, próceres de la independencia, sociedad republicana.

\section{REFLECTION ON THE POLITICAL, TERRITORIAL AND SOCIAL SITUATION OF PERU AFTER INDEPENDENCE}

Aвstract: This paper will critically address the process that led to our transition from colonial to republican society. For this, a systematic review of essential documents that influenced this process will be given, as well as the parallel of historical events given at the international level. This analysis will also take as a basis the history of political ideas, which influenced the thinking of the characters who helped build the politics and society that - abandoned the colonial model.

Keywords: History of Peru, independence of Peru, Peruvian viceroyalty, intellectuals of independence, republican society.

\section{Introducción}

71 presente trabajo es una reflexión sobre los principales aspectos políticos, territoriales y sociales, que se desarrollaron durante la transición colonial a la republicana de nuestra nación. Se buscará, de esta manera, proponer nuevas interpretaciones a los problemas básicos y generales que hasta el día de hoy se puede observar en nuestra sociedad. Siendo la segunda década del siglo XIX el inicio de nuestro sistema republicano 
como Nación, se detalla fechas y acontecimientos posteriores, inclusive del siglo XX, con el objetivo de poder comprender algunas situaciones actuales como ya se ha mencionado.

Para una mejor comprensión de las explicaciones y propuestas sobre el tema, se ha divido el presente trabajo en tres momentos. El primero corresponde a los antecedentes de la independencia peruana, en ella se enfatiza la importancia de la separación de las trece colonias norteamericanas del dominio inglés, asimismo, se reflexiona sobre los aportes de la Revolución Francesa para finalmente presentar las actividades inmediatas que se dieron en el Perú como consecuencia de los hechos anteriormente mencionados. El segundo momento comprende la presentación de la situación social que se vivía en los primeros años de la independencia, junto a ello, la ineficacia política de los sectores sociales privilegiados que conllevaron especialmente a una falta de defensa nacional, especialmente en el contexto territorial. Finalmente, en el tercer momento se presentan las conclusiones, las mismas que se consideran "no cerradas" y que son propuestas para nuevos temas de investigación. No es el interés del presente trabajo dar soluciones o respuestas universales a las situaciones que se dieron durante nuestro nacimiento como país supuestamente republicano, sino proponer temas de debate, especialmente si se considera que estamos cerca a celebrar nuestro Bicentenario. 


\section{Los Antecedentes de la Proclama Independentista}

\subsection{La Independencia de las trece colonias}

Cuarenta y cinco años antes de que el general San Martín proclamará la independencia peruana, Norteamérica será el escenario en donde se desarrollaría uno de los hechos históricos más importantes en los albores de la edad contemporánea: la independencia de las trece colonias inglesas, y por ende, el nacimiento de los Estados Unidos.

A diferencia del Perú, que era colonia española desde el siglo XVI, Norteamérica fue colonizada desde inicios del siglo XVII por ingleses de confesión puritana calvinista. Con el transcurso del tiempo se fueron formando trece colonias permanentes, las mismas que para el siglo XVIII alcanzaron un alto desarrollo económico rural (Haciendas), especialmente en la producción del azúcar y el tabaco.

Asimismo, las colonias participaban de sus propias decisiones políticas, unidas a la autoridad del monarca inglés. A diferencia del Perú, cada una de las colonias tenía su Asamblea Electa, que según Carlos Alvear:

[Eran] pequeños parlamentos, que desempañaron un gran papel en la historia política norteamericana, porque ayudaron a fortalecer el principio de que la autoridad [monarca] debe contar con el consentimiento del pueblo [colonias], y dieron, al mismo tiempo, oportunidad para que los hombres públicos norteamericanos se ejercitaran en funciones de gobierno, de tal modo que cuando se hizo la independencia contaban con experiencia adecuada. (2004, p.34) 


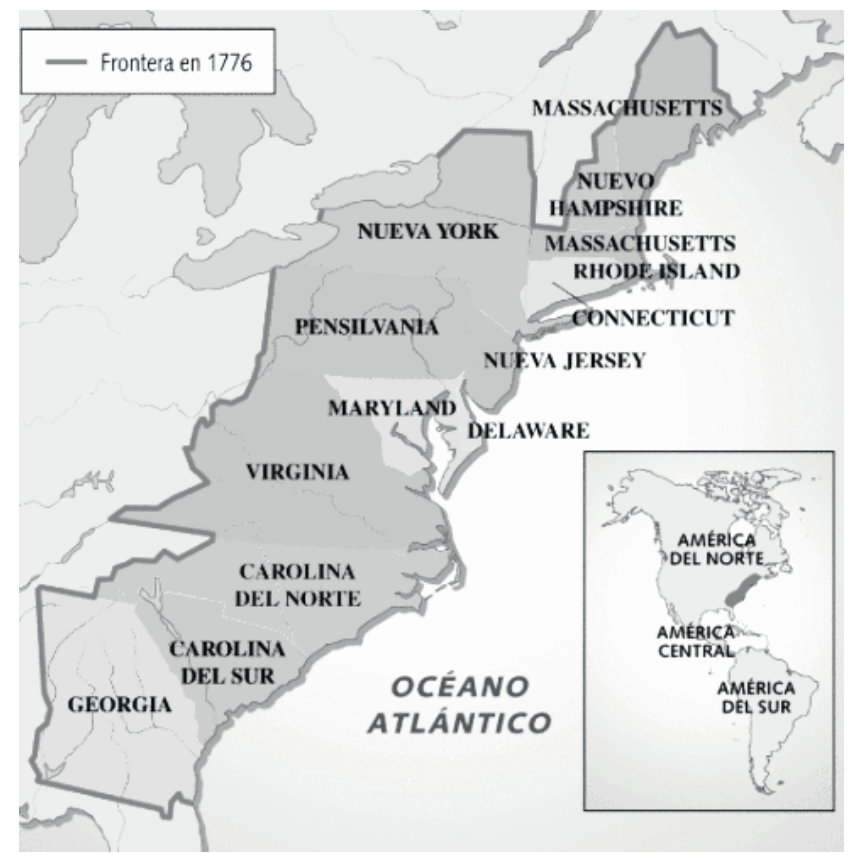

Figura 1. Mapa de las trece colonias en el año de 1776. Adaptado de "Historia Universal," por Santillana, 2006, La enciclopedia del estudiante, Tomo 2.

Cabe señalar, que aquellos "hombres públicos norteamericanos" a los que hace referencia Alvear, forman parte de una generación muy distinta a la que llegó inicialmente, y con justa razón son considerados como “criollos". Se trata de aquellos hacendados, intelectuales, pastores religiosos, militares, etc., nacidos en tierras americanas ${ }^{1}$.

1 Teniendo en cuenta que el primer asentamiento ingles en Norteamérica fue Jamestown y posteriormente la colonia de Virginia (1606 - 1607), se debe considerar a los participantes de la obra emancipadora, como "criollos", pues nacieron durante o, después de la fundación de dichos poblados, así tenemos a Benjamín Franklin (1706-1790), 
Las razones que dieron origen al proceso emancipador, descansan en las amenazas — por parte de la corona inglesa— a los intereses económicos de las colonias. Así tenemos, por ejemplo, la imposición de nuevos gravámenes (Ley del Timbre: 1765), leyes contra el comercio y la industria colonial, la restricción en la producción de azúcar y una política agresiva de monopolio.

Aunque el suceso inicial fue el motín del Té (Boston, 1773), los momentos importantes y decisivos se desarrollan a partir de los Congresos Continentales realizados en la ciudad de Filadelfia. Allí reunieron los delegados de las diversas Asambleas Electas.

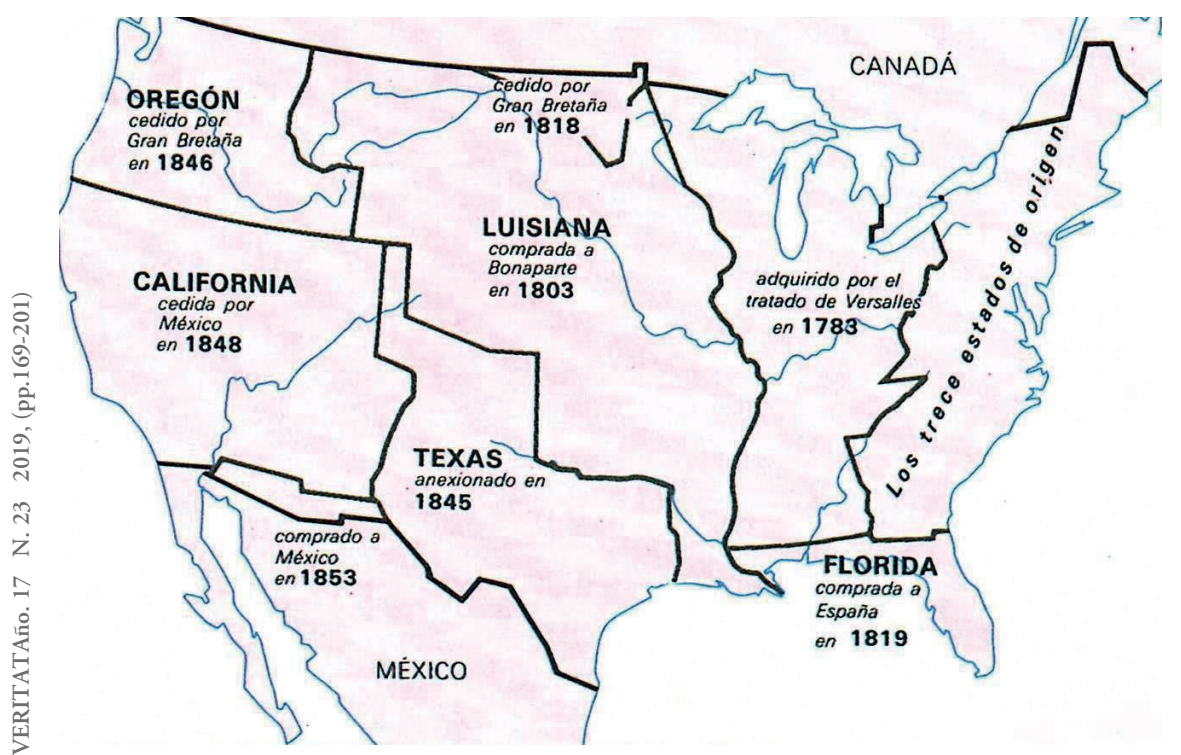

Figura 2. La ampliación territorial de los Estados Unidos. Adaptado de "Absolutismo e Ilustración,” por De Agostini, 2000, Historia Universal, Tomo 8.

John Adams (1735-1826), Thomas Jefferson (1743-1826), George Washington (17321799), Patrick Henry (1736-1799), entre otros. 
REFLEXIÓN SOBRE LA SITUACIÓN POLÍTICA, TERRITORIAL

Y SOCIAL DEL PERÚ DESPUÉS DE LA INDEPENDENCIA

En el primer Congreso Continental (1774), se redactó la Declaración del respeto a los derechos de las colonias. En el segundo Congreso Continental (1776), se proclama la Independencia de las trece colonias $^{2}$. Para 1783, la corona inglesa reconoce la Independencia de las colonias mediante el Tratado de Versalles. Finalmente, en el tercer Congreso Continental (1787), se promulgó la Constitución Norteamericana, así como también se definió el tipo de gobierno a seguir y la elección del primer presidente (Jorge Washington).

$\mathrm{La}$ independencia de las trece colonias, fue tomada como "modelo" para las demás realidades coloniales latinoamericanas. Era la primera separación de unas colonias de la administración imperial, y no de cualquier monarquía europea, sino, del imperio colonial más poderoso del mundo moderno como era Gran Bretaña. Unido a ello, la Constitución norteamericana — la única que sigue vigente hasta hoy_ "estableció una república democrática, representativa y federal, aunque respetándose los gobiernos locales de cada uno de los estados" (Alvear, 2004, p. 41), mediante la presencia del poder ejecutivo "federal" que era el Presidente, el poder legislativo representado en un Congreso bicameral (senadores y diputados) y en el poder judicial, que recaía en la Corte Suprema de Justicia. Finalmente, el historiador alemán Norbert Rehrmman $(2016)^{3}$ añade que, por parte de Estados Unidos, no se dio ninguna política de apoyo — especialmente militar - hacia los países latinoamericanos que se encontraban bajo dominio español, tal cual lo verificó Simón Bolívar en su visita a Estados Unidos en 1807. Argumenta el mencionado historiador,

2 Posterior a la proclama de la Independencia, se desarrollan las batallas finales y decisivas a favor de la causa americana: Saratoga (1777) y Yorktown (1781).

3 Véase Radio Programas del Perú Noticias (2016). 
que dicha postura se debió al apoyo que recibieron las colonias por parte de España $^{4}$, a favor de la independencia.

\subsection{La Revolución Francesa}

Trece años después de proclamarse la independencia de las trece colonias, y treinta y dos años antes de la independencia peruana, Europa volvía a ser escenario del segundo acontecimiento histórico que motivaría a las colonias hispanas en Latinoamérica a buscar el camino de la emancipación.

La Revolución Francesa tuvo como base ideológica las diversas corrientes filosóficas que se fueron desarrollando en la edad moderna: Racionalismo, Positivismo y la Ilustración. Todas ellas, llevadas al campo político que vivía la Francia borbónica de Luis XVI — por pensadores como Voltaire, Montesquieu y Rousseau-, llegaron a proponer la necesidad de un nuevo sistema de gobierno en donde la monarquía debía compartir el poder con el denominado Estado llano representado en Poderes; a ello se a fue uniendo factores como el debilitamiento del poder real, la necesidad de separar el poder religioso del político y la crisis financiera que atravesaba Francia.

En el caso de Francia, el grupo social que conllevó a la revolución fue el Estado llano, también denominado tercer estado, o simplemente la gran masa social francesa que estaba compuesta por el simple hombre de a pie, pasando por los profesionales hasta llegar a las grandes familias con poder económico denominadas burguesas. Todos ellos tenían algo en común: la

Fueron especialmente tres las monarquías europeas que ostentaban colonias en América: Gran Bretaña, España y Francia. Por tal motivo, cuando se daba una revuelta o movimiento emancipador en alguna de las colonias, las otras monarquías apoyaban dichos actos con el fin de quebrantar la hegemonía de la(s) otra(s). 
REFLEXIÓN SOBRE LA SITUACIÓN POLÍTICA, TERRITORIAL

Y SOCIAL DEL PERÚ DESPUÉS DE LA INDEPENDENCIA

ausencia de privilegios como sí los tenía la nobleza y el clero, consideradas como los otros estados.

El Estado llano a su vez estaba dividido en dos sectores o protopartidos políticos: los girondinos y los jacobinos. Los primeros estaban integrados por la burguesía que se ha mencionado anteriormente, los cuales buscaban limitar el poder real; mientras que los segundos, estaban compuestos mayoritariamente por profesionales que anhelaban imponer una república democrática.

A diferencia de las trece colonias, en Francia no se buscó una independencia, sino una revolutio, esto significa, una vuelta, un cambio, una reforma, sin necesidad de una separación o independencia de "las instituciones políticas, económicas o sociales de una nación" (RAE, 2005, p.1338). Así lo demuestran las primeras etapas de la Revolución Francesa, tanto en la Asamblea Constituyente (1789-1791) como en la Asamblea Legislativa (1791-1792) el poder monárquico es limitado pero no extinguido; lo que sí se dará en la Convención Nacional (1792-1795) y en el Directorio (1795-1799) hasta la llegada de Napoleón al poder (17991815).

Contrario a la noticia de la independencia de las trece colonias, el conocimiento de la Revolución Francesa fue "universal". Esto debido a que se dio en la propia Francia, cuna del denominado Absolutismo monárquico (Antiguo Régimen), descalificando este tipo de gobierno y confirmando el sistema republicano que ya se daba en los Estados Unidos.

Para los criollos latinoamericanos —especialmente en el Virreinato del Perú - la actuación del Estado llano francés, fue un claro ejemplo

5 El conocimiento de la caída del Antiguo Régimen y las nacientes propuestas políticas en Europa, fueron de conocimiento, especialmente de las elites criollas intelectuales - tanto laicos como religiosos - a partir de los documentos y libros que llegaban clandestinamente al puerto del Callao, como a los viajes que realizaban estos a los países europeos. 
de cómo alcanzar el poder político, al cual estaban ausentes. Esto queda fundamentado con la difusión de los principios de la Igualdad ${ }^{6}$, Fraternidad y Libertad.

\subsection{Los movimientos reformistas y separatistas en el Virreinato peruano}

En el caso del Perú de mediados del siglo XVIII, la independencia de las trece colonias y la Revolución Francesa, influenciaron en los criollos - especialmente intelectuales y profesionales - quienes gozaban de respeto y admiración dentro de la sociedad colonial (prócer) e iniciaron todo un conjunto de reflexiones políticas con miras a aplicarlas a un futuro inmediato (precursores).

Al igual que la Francia revolucionaria, en el Perú se formaron dos posturas políticas frente al poder español. En primer lugar se encontraban aquellos criollos precursores denominados reformistas o fidelistas, quienes buscaban modificar algunas instituciones políticas dentro del Virreinato peruano, participar del gobierno, pero no desligarse de la corona española.

Entre estos personajes tenemos al poco conocido Fray Calixto de San José Túpac Inca (1710-1782), religioso mestizo que viaja a España en 1750 y expone ante la corona española su "Exclamación Reivindicacionista",

6 Cabe señalar que el principio de Igualdad, fue más teórico que práctico durante la revolución. Citemos, por ejemplo, la condición de la mujer durante este proceso, inicialmente fue reconocida como ciudadana, pero con el transcurrir de los años de la primera república, la mujer tuvo que luchar para conseguir nuevamente este reconocimiento como ciudadana. Un claro ejemplo de esta lucha por la igualdad es Marie Gouze (Olympe de Gauges), quien en 1791 publicó a manera de protesta, la Declaración de los derechos de la mujer y de la ciudadana.

7 Teniendo en cuenta que su exclamación se realiza en 1750, dicho personaje es considerado como el primer precursor "reformista", no solo del Perú, sino de toda la América. 
REFLEXIÓN SOBRE LA SITUACIÓN POLÍTICA, TERRITORIAL

Y SOCIAL DEL PERÚ DESPUÉS DE LA INDEPENDENCIA

mediante la cual solicita la eliminación de la Mita y los Corregimientos, lamentablemente es recluido y muere en la cárcel. Otro conocido personaje es José Hipólito Unanue y Pavón (1755-1833), médico de profesión, que con el pseudónimo de Ariosto, escribió en el Mercurio Peruano su postura de imponer una monarquía constitucional, ideal que fue compartido con el general San Martín ${ }^{8}$. El ilustre abogado José Baquijano y Carrillo (17511817), quien con motivo de la llegada del virrey Jáuregui (27 agosto 1871), dio el discurso de bienvenida conocido como el "Elogio a Jáuregui" que se caracterizó por ser una protesta sobre los abusos del sistema virreinal y la sustentación que la condena a muerte de Túpac Amaru II fue ilegal. Finalmente, pero no menos importante, se encuentra el religioso Toribio Rodríguez de Mendoza (1750-1825), quien en su función de rector del Real Convictorio de San Carlos, impuso muchas enseñanzas tomadas de la Ilustración.

La segunda postura era la denominada separatista o independentista, integrada por aquellos hombres que buscaban la separación definitiva de la corona española. Dos personajes significativos de esta postura son José de la Riva-Agüero y Sánchez Boquete (1783-1858) y Mariano José de Arce Bedrigal (1782-1852). El primero — quien llegará a ser el primer presidente del Perú- escribió en 1818 su "Manifestación Histórica y Política de la Revolución de América”, conocida también como las "28 Causas”. En ella presentaba 28 razones por las cuales se justificaba la sublevación de América frente a todo régimen colonial y la búsqueda de la Independencia. El segundo, fue un religioso arequipeño que a través de sus predicas declaraba

8 Su actividad política es cuestionada, pues se adhirió a cuatro momentos políticos de nuestro país. Inicialmente estuvo cercano al gobierno de los dos últimos virreyes del Perú (Abascal y Pezuela), por otra parte lo encontramos como ministro en el protectorado del general San Martín y luego como presidente del consejo de gobierno de Simón Bolívar; en términos modernos un "tránsfuga". 
al Rey Fernando VII como usurpador y acusaba a la conquista como causa de la pobreza e ignorancia de América, de la cual tenía que salir mediante la independencia de los pueblos.

A inicios del siglo XIX, la actividad de los precursores va a ir acompañada de diversos movimientos de sublevación, los cuales se darán especialmente por intelectuales, políticos y militares provincianos.

Se cita por ejemplo, el Plan Subversivo de José Aguilar y Manuel Ubalde (Cuzco, 1805), que se resume en el anhelo de restaurar el incanato, un tipo de mesianismo andino que se inicia con la toma del cuartel del Cuzco, pero desafortunadamente no progresa. Seis años después se da la Revolución de Francisco de Zela (Tacna, 1811), quien se proclama "Comandante militar de las milicias de América" después de tomar los cuarteles militares de Tacna. Es apresado y muere en el destierro. Al año siguiente (1812), se da la Revolución del corregidor Juan José Crespo y Castillo (Huánuco), quien es proclamado como caudillo, después de la toma de la ciudad (12 febrero) por los nativos, hasta que fueron derrotados en la batalla de Ambo (17 marzo). Finalmente, la Sublevación de los Hermanos Angulo y Pumacahua (Cuzco, 1814) es considerada como la mejor organizada, gracias al ejército dividido en tres columnas (Puno-Alto Perú, Arequipa y Huamanga), lamentablemente todas fueron derrotadas.

Ninguna de las sublevaciones provinciales logró sus objetivos, y esto gracias a la política administrativa y militar llevada a cabo por el penúltimo virrey del Perú: José Fernando de Abascal y Sousa. Durante el periodo comprendido entre 1806 hasta 1816, interfirió en todo acto emancipador de nuestro territorio. El Perú tuvo que esperar la llegada de la Corriente Libertadora del Sur dirigida por el general don José de San Martín, para que el sábado 28 de julio de 1821 se proclamara la independencia del Perú y, de 
REFLEXIÓN SOBRE LA SITUACIÓN POLÍTICA, TERRITORIAL

Y SOCIAL DEL PERÚ DESPUÉS DE LA INDEPENDENCIA

esta manera, se iniciara todo un proceso que finalizará mediante la guerra con España en 1866, con el victorioso combate del 2 de mayo?.

\section{El Perú después de la Proclama Independentista}

\subsection{El aspecto político: de los sectores políticos al caudillismo inicial}

Durante los primeros años de la Independencia, la búsqueda de una forma de gobierno en el Perú, se basó en tres grandes posturas. La primera fue propuesta por el general San Martín, quien insistía en la urgencia de establecer una Monarquía Constitucional. Por su parte, Simón Bolívar proponía una Dictadura de corte vitalicia.

Ante estas posturas, se abría paso una nueva ideología — que tenía como modelo a la revolución francesa- de tendencia liberal (separatista, independentista), que afirmaba que el Perú se encontraba ya listo para adoptar el modelo Republicano.

Por esto, se afirma que el Perú fue fundado bajo la base de la razón y de los ideales de justicia e igualdad social, pues sus seguidores - entre ellos, José Faustino Sánchez Carrión y Francisco Javier de Luna Pizarro- dieron al país una Constitución de tipo republicano. Esta reconocía la existencia de un Congreso bicameral, ambas cámaras elegidas y reconocidas por las

9 En 1863, la corona española busca restaurar su política colonial en América, por tal motivo envía una supuesta "Expedición científica española", dándose en el Perú el incidente de la hacienda Talambo, que será el inicio del conflicto. Después del Combate del 2 de mayo (1866), Espańa renuncia a todo tipo de intervención en los nuevos Estados americanos. 
masas; junto a ello, la persistencia de un conjunto de garantías sociales e individuales traducidas en deberes y derechos.

Lamentablemente, en los primeros años del Perú independiente, no se produjeron los cambios que se esperaban, especialmente en lo referente a la integración social. Perduraban aún las formas de dominio colonial: no se podía hablar todavía de un país compuesto de ciudadanos criollos, mestizos, indios, etc., que dirigieran conjuntamente la política y la economía del país.

El Perú se encontraba hasta cierto punto, en manos de una oligarquía, una minoría que no pudo consolidarse en el poder, pues su preocupación como grupo seguía siendo conservar sus privilegios heredados. Las consecuencias de esta actitud fueron desastrosas para nuestro país durante los primeros años de su independencia, pues existía por parte de esa elite en decadencia, una pronunciada indiferencia frente a los problemas políticos, económicos y sociales, que afectaba a toda la nación. La unidad nacional se convirtió simple y llanamente en una utopía que no podía resolver los desafíos derivados de la existencia de varias culturas o realidades sociales en un mismo territorio. A pesar de ello, este grupo social pugnó por dirigir la política de nuestro país. Su posición se denomina conservadora (reformista o fidelista).

Pero, después de la proclama independentista, ¿cuáles eran las nuevas diferencias entre los liberales y los conservadores? Liberales como Sánchez Carrión y Luna Pizarro, consideraban que el Gobierno debía descansar en una ruptura del centralismo administrativo; la necesidad de la separación de la Iglesia y el Estado; la subordinación del ejército al poder político y la promoción de gobiernos locales e independientes, entre otros. Muy contrario a ello, la postura conservadora, que tuvo como principal representante a Bartolomé Herrera, consideraba la importancia de un Estado centralista, en 
donde el soberano ejerce el poder por mandato divino; la participación de la Iglesia y el ejército en la política del país, así como la importancia de los principios morales en el orden y la unidad de la sociedad.

Lamentablemente la presencia de ambas posturas políticas e ideológicas, acabaron haciendo que el Perú se convirtiera en un escenario de lucha entre ambos sectores políticos, que al no encontrar una solución inmediata, hicieron partícipes también de dicha contienda a los militares (caudillos), quienes al final ostentaron el poder, apoyados por los anteriores. De esta manera, el Perú nace como un Estado empírico, afectado por un gran abismo social, tal como lo refiere el historiador Jorge Basadre, a diferencia de nuestro vecino país de Chile.

La denominación de caudillismo, fue acuñada por el historiador tacneño Jorge Basadre. Por medio de este término quiso hacer referencia a tres momentos ${ }^{10}$ durante los cuales el control del Estado peruano se encontraba en manos de los militares.

Como se ha mencionado anteriormente, a la falta de una sólida clase política, capaz de asumir el control del Estado en los primeros años de nuestra República, la única salida para este trance fue la presencia de los militares en el poder. Como refería José Carlos Mariátegui (2005), eran los únicos que podían garantizar un régimen mediante las "armas" y porque gozaban de prestigio por sus "glorias" en los conflictos bélicos durante la Independencia.

El caudillismo no fue un fenómeno único en el Perú, sino que se dio en toda Latinoamérica durante el siglo XIX. Así, por ejemplo, en México

10 Denominados militarismos por el mismo historiador, el primer militarismo comprende después de la Independencia, el segundo militarismo abarca los gobiernos posteriores a la Guerra con Chile y, finalmente, el tercer militarismo se da en diversos momentos de la historia peruana del siglo XX. 
se desarrolló un caudillismo rural; en Venezuela y Argentina se presentó un caudillismo pampeano y en Bolivia uno campesino. El caudillismo peruano fue siempre militar, tal como se puede constatar desde el general San Martín hasta el gobierno del coronel José Balta.

Una característica del caudillismo lo constituyeron las grandes revueltas y golpes de Estado que se dieron entre los propios militares, apoyados por los sectores liberales y conservadores. Cabe mencionar, por ejemplo, el gobierno de Agustín Gamarra (1829-1833), quien tuvo que afrontar 17 golpes de Estado o conspiraciones.

Por otra parte, durante este lapso, los militares siguieron los ideales de los libertadores, esto significó la presencia de gobernantes prosanmartinianos y probolivarianos. Entre estos últimos podemos mencionar al militar Andrés de Santa Cruz, quien durante su gobierno (1836-1839) procedió a organizar la Confederación peruano-boliviana. 
REFLEXIÓN SOBRE LA SITUACIÓN POLÍTICA, TERRITORIAL

Y SOCIAL DEL PERÚ DESPUÉS DE LA INDEPENDENCIA

Tabla 1

Reglamentos, Estatutos, Pactos y Constituciones del Perú Republicano (18211867)

\begin{tabular}{|c|c|c|c|c|c|}
\hline Constitución & Fecha & Régimen & Numerales & Clasificación & Vigencia \\
\hline \multicolumn{5}{|c|}{ - Reglamento Provisorio - promulgado por San Martin - 12/02/1821 } & 9 meses \\
\hline \multicolumn{2}{|c|}{ - Estatuto Provisorio } & \multicolumn{3}{|c|}{ - promulgado por San Martin $\quad$ - 08/10/1821 } & 1 aก็่ \\
\hline \multicolumn{5}{|c|}{ - Bases de la Constitución - promulgado por el Congreso - 17/12/1821 } & 11 meses \\
\hline $1^{\circ}$ & $12 / 11 / 1823$ & Congreso & 194 Art. - secciones & Liberal & Suspendida \\
\hline $2^{\circ}$ & $09 / 12 / 1826$ & Santa Cruz & 150 Art. - 11 títulos & Cesarista & 49 días \\
\hline $3^{\circ}$ & $18 / 03 / 1828$ & La Mar & 182 Art. -10 títulos & Liberal & 6 aก̆os, 3 meses \\
\hline $4^{\circ}$ & $10 / 06 / 1834$ & Orbegoso & 187 Art. - 11 títulos & Liberal & 5 аต̆оง. 2 meses \\
\hline \multicolumn{5}{|c|}{ - Pacto de Tacna - promulgado por Santa Cruz - 09/05/1837 } & 1 аศ̆о, 8 meses \\
\hline $5^{\circ}$ & $10 / 11 / 1839$ & Congreso & 193 Art. - 19 títulos & Conservador & 15 años, 8 meses \\
\hline \multicolumn{5}{|c|}{ - Estatuto Provisorio - promulgado por Ramón Castilla-17/07/1855 } & 1 año, 3 meses \\
\hline $6^{\circ}$ & $19 / 10 / 1856$ & Castilla & 140 Art. -19 títulos & Liberal & 4 ańos, 1 mes \\
\hline $7^{\circ}$ & $13 / 11 / 1860$ & Castilla & 138 Art. - 19 títulos & Moderado & 7 ап̆ов, 2 meses \\
\hline $8^{\circ}$ & $12 / 08 / 1867$ & Prado & 131 Art. - 19 títulos & Liberal & 4 meses, 8 días \\
\hline
\end{tabular}

Nota. De las doce Constituciones que ha tenido el Perú durante toda su vida republicana, ocho corresponden al periodo que comprende las seis décadas posteriores a la proclama de la Independencia. La redacción de varias Constituciones confirma la necesidad por parte de los militares de legitimar sus gobiernos y consecuentemente sus actos.

En conclusión, el caudillismo también denominado Primer Militarismo, no aportó mucho al ideal del Perú republicano, ya que también ingresó a un período de inestabilidad política; causado por los propios intereses personales de los militares, quienes antes de fortalecer la política del Estado e integrar a la sociedad, no hicieron otra cosa que mantener a las clases sociales fuera del control político. Es así como el historiador arequipeño Eusebio Quiroz Paz-Soldán (1989) afirma que: 
Mientras en el Perú no apareció un grupo civil que gobernará hasta 1870. Entre 1831 y 1879 Chile tuvo seis presidentes constitucionales. En el Perú, en el mismo período hubo hasta veinte gobernantes. En Chile se realizó un proceso de afianzamiento institucional y de estabilidad gubernativa que lo condujo a la unidad; la situación en el Perú no era la misma. (p. 15)

\subsection{El aspecto territorial: de la unidad al desmembramiento}

Desde el año 1784 el virreinato del Perú comprendía territorialmente ocho Intendencias, al respecto, el historiador Del Busto (2004) señala que el nombre "proviene del latín intendens e intendentis, derivado de intendere, que significa 'dirigir', 'encaminar'. Fue un cargo de gobierno que se [impuso] para reemplazar (...) sobre todo a los corregidores” (p.105). La Intendencia es un claro ejemplo de las reformas borbónicas promovidas por el rey Carlos III. Al ser una institución política de origen medieval francés, dieron buenos resultados en la organización política de los virreinatos hispanos en América. Tanto así que las funciones del Intendente eran iguales, pero en poca proporción a las del virrey (Del Busto, 2004). 
REFLEXIÓN SOBRE LA SITUACIÓN POLÍTICA, TERRITORIAL

Y SOCIAL DEL PERÚ DESPUÉS DE LA INDEPENDENCIA

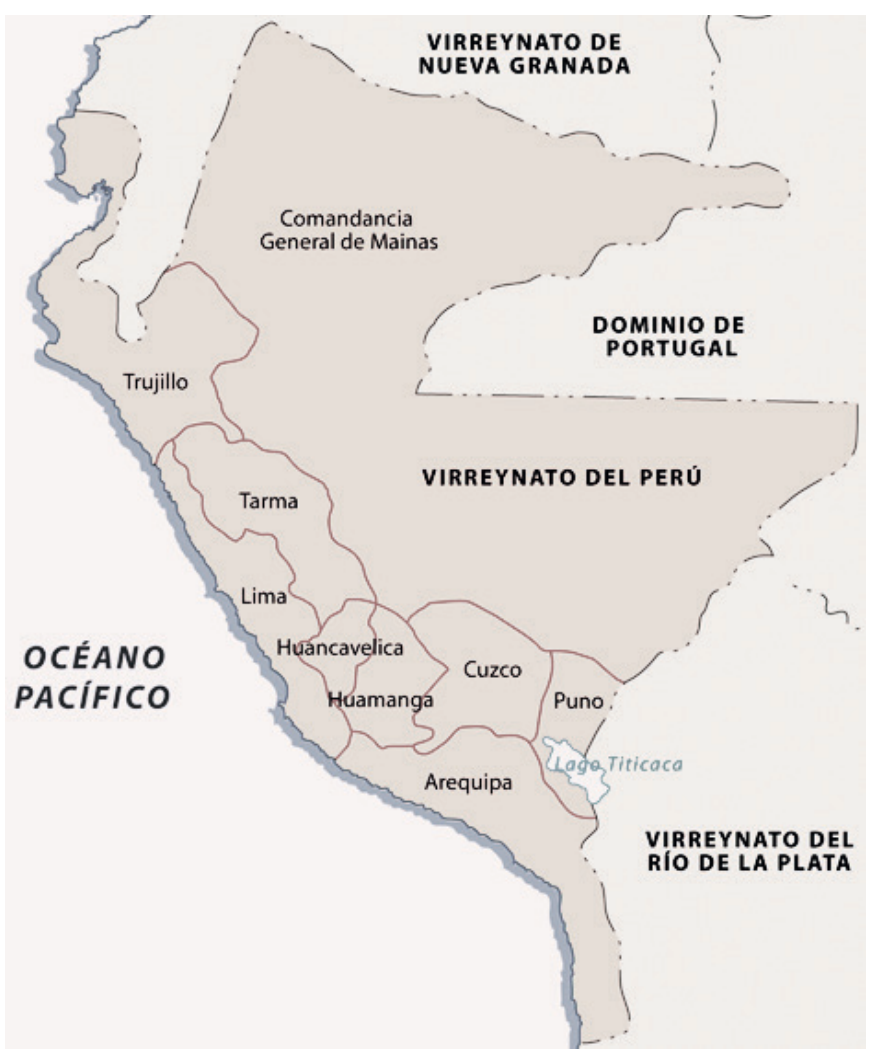

Figura 3. El Virreinato del Perú y sus Intendencias a inicios del siglo XIX. Adaptado de "Conquista y Virreinato," por J. Del Busto, 2004, Enciclopedia Temática del Perú (T. II).

El virreinato del Perú comprendía ocho Intendencias: Trujillo, Lima, Tarma, Huancavelica, Huamanga, Arequipa, Cuzco y Puno. Estas se dividían a su vez en Partidos judiciales o simplemente Partidos ${ }^{11}$, cada uno de ellos administrados por un subdelegado.

11 Al iniciarse el siglo XIX, el virreinato del Perú comprendía 56 partidos. 
Esta división territorial colonial será modificada seis meses antes de proclamarse la Independencia del Perú. Primero, mediante los Reglamentos Provisorios del 12 y 17 de febrero de 1821 dados en Huaura, y posteriormente mediante el Decreto del 26 de abril de 1822 — todos ellos propuestos por el general San Martín-. De esta manera, las antiguas Intendencias españolas se convirtieron en Departamentos y los Partidos en Provincias, siendo posteriormente reconocidas mediante la Constitución de 1823:

El Congreso fijará los límites de la República, de inteligencia con los Estados limítrofes, verificada la total independencia del alto y bajo Perú. Se divide el territorio en departamentos, los departamentos en provincias, las provincias en distritos, y los distritos en parroquias. (Capítulo II, Territorio Artículo $6^{\circ}$ y $7^{\circ}$ )

Mediante los Reglamentos Provisionales del 12 y 17 de febrero de 1821, surgen los tres primeros departamentos: La Libertad ${ }^{12}$, que comprendía la anterior intendencia de Trujillo y la Comandancia General de Maynas y Jaén, y abarcaba diez provincias. Ayacucho ${ }^{13}$, correspondiente al territorio de las antiguas intendencias de Huamanga y Huancavelica, debía su cambio de nombre a la victoriosa Batalla de Ayacucho, y abarcaba nueve provincias. Finalmente, Junín ${ }^{14}$ es creado tomando como base el territorio de la anterior

12 Se cambió el nombre de Trujillo a la Libertad para perpetuar la memoria de haberse organizado allí la campaña final de la Independencia, reconocimiento que fue hecho mediante Decreto del 9 de marzo de 1825.

13 El cambio de nombre del departamento se oficializa a partir del Decreto del 15 de febrero de 1825

14 El cambio de nombre del departamento se oficializa a partir del Decreto del 3 de setiembre de 1825 . 
intendencia de Tarma. De la misma manera, su nombre se debe a la batalla de Junín; abarcaba ocho provincias.

La importancia del departamento de Lima radica en que en ella se encontraba la ciudad del mismo nombre, desde donde se centralizaba el poder político que se había heredado desde la fundación de la misma (1535) y su confirmación real como capital del Virreinato (1542). Reconocimiento que es confirmado mediante el Decreto Supremo del 4 de agosto de 1821.

Finalmente, los tres siguientes departamentos son creados mediante Decreto del 26 de abril de 1822: Cuzco que comprendía once provincias, Arequipa con siete provincias y Puno con cinco provincias. Todas ellas llevarían el mismo nombre de cuando eran Intendencias.

Lamentablemente, aquel territorio que se heredó del sistema colonial y se modificó en departamentos, aplicándose el principio del Uti Possidettis ${ }^{15}$ ("como poseéis de acuerdo al derecho, así poseeréis"), iba a ser desmembrado cinco décadas después de la proclama independentista hasta mediados del siglo XX.

Aunque entre las principales causas de este desmembramiento territorial se encuentra la política expansionista de los países vecinos, no cabe duda que la ineficacia de los gobiernos del primer militarismo y los siguientes gobiernos civiles, reflejados en la ausencia de planes de resguardar las fronteras, — peor aún— en los errores diplomáticos al negociar, redactar o firmar tratados internacionales, hicieron que el Perú perdiera gran parte de su territorio inicial.

15 Es un principio legal que proviene del Derecho Romano, en sus inicios justificaba el derecho a ciertos territorios que habían sido conquistados, especialmente a la fuerza. Para el siglo XIX, fue retomado por los nuevos Estados que salían del colonialismo y buscaban una demarcación territorial a partir de lo que habían sido anteriormente. 
De la misma opinión es el jurista peruano Héctor Vargas (2010), quien en el Proemio de su libro sobre los Tratados realizados por el Perú ${ }^{16}$ afirma que:

La historia de los tratados en el Perú es verdaderamente inquietante, más que eso, penosa, porque con ninguno de ellos la Nación ha salido victoriosa. De la sola lectura de sus textos y de la manera como se condujeron sus firmantes, es fácil comprobar que al otorgarlos no hubo indispensable amor patriótico para evitar la mutilación del territorio. (...) Más condenable aún, que tratando de justificar lo injustificable invocaban al antipatriótico argumento, que de las tierras que cedían eran suelos inhóspitos, bosques deshabitados, sin importancia y sin valor. [referente a la Amazonía] (...) Los textos de tan censurables instrumentos, que incrementan el Archivo Nacional, son verdaderos baldones que hieren el Honor Nacional. (pp. 14-15)

16 Jurista y político, Héctor Vargas Haya ha realizado un serio trabajo correspondiente a los 25 tratados, 3 convenios y 2 protocolos celebrados por el Perú, desde que nació como república hasta el primer gobierno de Alberto Fujimori. Véase las referencias bibliográficas al final del texto. 
REFLEXIÓN SOBRE LA SITUACIÓN POLÍTICA, TERRITORIAL

Y SOCIAL DEL PERÚ DESPUÉS DE LA INDEPENDENCIA

Tabla 2

Principales Tratados, Convenios y Protocolos Firmados por el Gobierno Peruano (1851-1942)

\begin{tabular}{|c|c|c|c|}
\hline País & Tratado/ Convenio/ Protocolo & Espacio perdido & Gobierno peruano \\
\hline \multirow[t]{3}{*}{ Brasil } & $\begin{array}{c}\text { Tratado Herrera - Da Ponte Ribeyro } \\
\text { (23 oct. } 1851)\end{array}$ & $56,000 \mathrm{~km}^{2}$ & José R. Echenique \\
\hline & $\begin{array}{c}\text { Tratado Velarde - Rio Branco } \\
\text { (8 set. 1909) }\end{array}$ & $169,000 \mathrm{~km}^{2}$ & 1. ${ }^{\text {er }}$ Gob. de Augusto B. Leguía \\
\hline & Tratado Muñoz - Netto & & \\
\hline & (27 marzo 1867) & $45,000 \mathrm{~km}^{2}$ & $1{ }^{\text {er }}$ Gob. de Mariano I. Prado \\
\hline Bolivia & $\begin{array}{l}\text { Tratado Muñoz }- \text { Netto } \\
\quad(27 \text { marzo } 1867)\end{array}$ & $99,000 \mathrm{~km}^{2}$ & 1. ${ }^{\text {er }}$ Gob. de Mariano I. Prado \\
\hline \multirow[t]{3}{*}{ Colombia } & Tratado Salomón-Lozano & & \\
\hline & (24 marzo 1922) & $122,000 \mathrm{~km}^{2}$ & 2. ${ }^{\circ}$ Gob. de Augusto B. Leguía \\
\hline & Apropiación de la zona de Quijos & $111,000 \mathrm{~km}^{2}$ & 2. ${ }^{\circ}$ Gob. de Mariano I. Prado \\
\hline Ecuador & $\begin{array}{l}\text { Protocolo de Rio de Janeiro } \\
\text { (29 enero 1942) }\end{array}$ & $50,000 \mathrm{~km}^{2}$ & 1. ${ }^{\text {er }}$ Gob. de Prado Ugarteche \\
\hline Chile & $\begin{array}{c}\text { Tratado de Ancón } \\
\text { (20 oct. 1883) } \\
\text { Tratado Rada - Figueroa } \\
\text { (3 junio 1929) }\end{array}$ & $64,000 \mathrm{~km}^{2}$ & $\begin{array}{c}\text { Gob. de Miguel Iglesias } \\
2 .^{\circ} \text { Gob. de Augusto B. Leguía }\end{array}$ \\
\hline
\end{tabular}

Nota. En la tabla se observa algunos de los tratados que ha celebrado el Perú con sus países vecinos, siendo el territorio más extenso en perderse la región amazónica, pasando a ser territorio brasileño. 


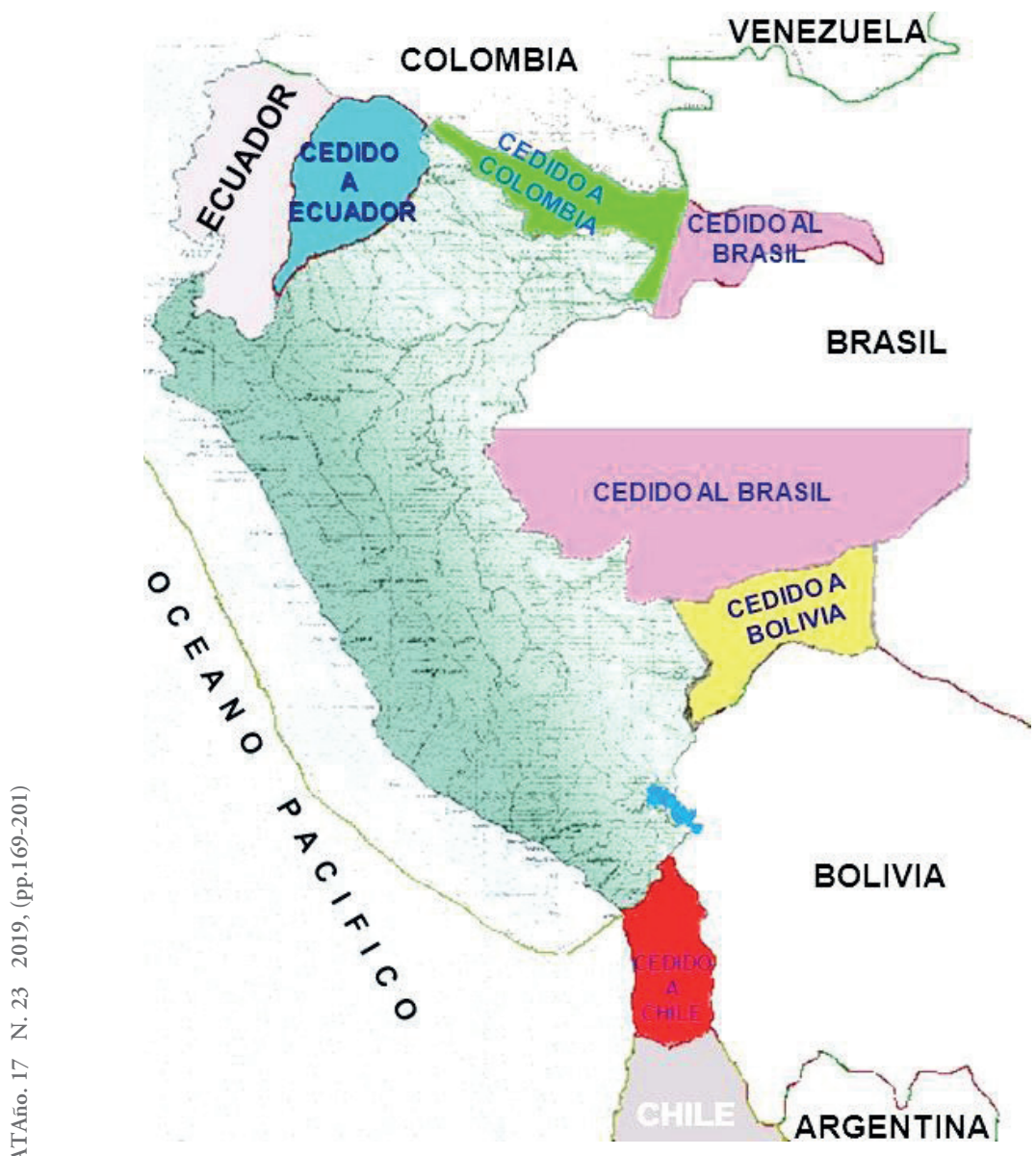

Figura 4. Territorios cedidos por el Perú por actos diplomáticos. Adaptado de "El Estado peruano 1. Realidad Nacional. El Estado: sus componentes, sus fines, sus instrumentos y sus medios. Bienestar Seguridad General Integral" por F. Villarrubia Marcelo, 2018. Recuperado de https://slideplayer.es/slide/11808560/ 
REFLEXIÓN SOBRE LA SITUACIÓN POLÍTICA, TERRITORIAL

Y SOCIAL DEL PERÚ DESPUÉS DE LA INDEPENDENCIA

Solamente queda comparar cuánto se ha perdido en soberanía territorial. Actualmente el territorio peruano comprende 1 '285,216 km². Al iniciarse como república en 1821, abarcaba aproximadamente 2’176,216 $\mathrm{km}^{2}$. A vísperas de celebrar el bicentenario de la independencia, se ha perdido aproximadamente unos $891,000 \mathrm{~km}^{2}$, lo que equivale a un $40,49 \%$ del total del área inicial.

Probablemente - a modo de justificación — se puede comprender las pérdidas territoriales que tuvo el Perú al inicio de su vida republicana. Así, los historiadores Carlos Contreras y Marcos Cueto (2000) señalan "que entre 1820 y 1850 el mapa político de América Latina sufrió severas transformaciones, al punto que nadie podía vaticinar cual sería el definitivo. [Por ejemplo,] México ${ }^{17}$ perdió casi la mitad de su territorio del norte, que pasó a manos de los Estados Unidos”18 (p. 29). Apreciación que no puede ser aplicada a las pérdidas territoriales posteriores a 1850 .

\subsection{El aspecto social: crisol de culturas}

Ahondar en el tema de la sociedad o sociedades que integran el Perú desde su independencia sería amplio o no iría con la naturaleza del presente artículo. Por un motivo didáctico se presentará las apreciaciones generales sobre los grupos sociales que vivieron la transición del sistema de gobierno virreinal al republicano, buscando identificar las principales características —más no las únicas_ que las diferenciaron entre ellas mismas.

17 Desde tiempos prehispánicos, los territorios de las futuras repúblicas de Perú y México, han compartido la importancia geopolítica de haber sido centros de formación de culturas avanzadas o grandes civilizaciones. De ahí la importancia de los españoles de fundar los dos principales virreinatos hispanos en América Latina en dichos territorios.

18 Véase la Figura 2 sobre la ampliación territorial de los Estados Unidos. 
La presencia española (godos o chapetones) continuó discretamente en la sociedad, tanto urbana como rural, después de 1821. Para el caso de Lima, la capital de la naciente república, señala Nelson Manrique (2004) que "de los 10 mil españoles que radicaban en Lima (...) quedaron apenas 600 (...) se produjo así la quiebra de la elite comercial y financiera que organizaba la economía colonial” (p. 15).

Inclusive, la presencia española es legitimada mediante la Capitulación de Ayacucho (1824), en donde se reconoce la presencia libre de los españoles —especialmente militares — en territorio peruano. Asimismo, se establece la paga por parte del nuevo Estado del viático de aquel español que decidía regresar a Europa.

Por lo anteriormente expuesto, el español podía vivir sin ninguna dificultad en el Perú, desarrollando las actividades que realizaba antes de la proclama, excepto los cargos políticos. Podía ser desde propietario de algún medio de producción hasta un simple trabajador de hacienda, conviviendo ${ }^{19}$ con las demás clases sociales.

Por su parte, los criollos (ahora denominados blancos), representaban a inicios de la república el 10 \% de la población en su totalidad. Descendientes de españoles, pero nacidos en el Perú, su característica primordial era ser el grupo social — aparte de los españoles — alfabeta, esto significa que sabían leer y en la mayoría de los casos también escribir. De aquí que la gran mayoría de criollos participantes del movimiento independentista venían del mundo urbano intelectual, seguido del comercial; a diferencia de los criollos del mundo rural quienes sobresalieron especialmente en el campo minero y las haciendas.

19 Un claro ejemplo de la convivencia entre "peruanos" y españoles, a mediados del siglo XIX, y los conflictos que se daban entre los mismos, es el incidente de la Hacienda Talambo ocurrido en agosto de 1863 . Véase la nota de pie de página número 9. 
REFLEXIÓN SOBRE LA SITUACIÓN POLÍTICA, TERRITORIAL

Y SOCIAL DEL PERÚ DESPUÉS DE LA INDEPENDENCIA

De esta manera, en la organización del Perú republicano, lograron alcanzar cargos en el ámbito castrense, religioso y especialmente político. De ese modo llegaron a ser, junto a los caudillos, los principales responsables de las decisiones políticas del Perú durante las siguientes décadas del siglo XIX.

A la clase criolla le seguía la indígena (habitante de un lugar) o andina (relacionado a los Andes), la misma que había aumentado después de la mortalidad dada especialmente en los siglos XVII y XVIII a causa de la Mita minera. No formaba un grupo homogéneo, sino que al igual que en la actualidad, comprendía un bagaje de subculturas que para inicios de la República tenían solo una cosa en común: comunicarse en diversas lenguas y dialectos, excepto en castellano.

Según Contreras y Cueto (2000) la postura del andino frente a los primeros gobiernos de la República fue de indiferencia, esto debido a que no se sentían identificados con las nuevas autoridades - tanto centrales como regionales - que se les imponía. Señalan los mismos historiadores que inclusive algunas comunidades pusieron resistencia para pasar a formar parte de la administración republicana, como fue el caso de la zona de Iquicha, al norte de Ayacucho entre 1824 y 1830.

Con el transcurrir de los años, fortalecieron su presencia en la sierra, a partir de comunidades agrarias a la manera del mundo de los Incas (especialmente Ayni y Minka), aunque hubo también significativamente presencia andina en las haciendas, cumpliendo la función de siervos (Yanaconaje).

$\mathrm{El}$ andino pasó gran parte del siglo XIX viviendo en la ignorancia o indiferencia de la actividad política del país, acaso, como señala Manrique (2004), por causa del analfabetismo que imperaba en los departamentos del Perú. Otra causa seria el no cumplimiento de la abolición del tributo 
que venía desde el general San Martín, como obra inconclusa de su protectorado. El andino de mediados del siglo XIX, no concebía el concepto de nacionalidad, sino por el contrario, se identificaba con personajes que hacían referencia a aspectos históricos-culturales ancestrales. Un claro ejemplo se encuentra en el contexto de la Guerra de 1879, el historiador Wilfredo Kapsoli, refiriéndose a una memoria de doña Antonia Moreno, señala que:

Para los indios Cáceres era de reencarnación Inca, por eso se postraban delante de él. Ellos creían que Cáceres era el continuador de sus antiguos señores los Incas y, siempre que lo veían le querían rendir homenaje, mezcla de cariño y gratitud. Le llamaban "Tayta” (padre, señor). (1995, p. 58)

El hombre andino, tuvo que esperar hasta la segunda década del siglo XX, para ser reconocido, a partir de las comunidades campesina, mediante las Constituciones que aparecen durante el Oncenio de Leguía. Unido a ello en el campo cultural se da el movimiento indigenista, el mismo que resalta el aporte del hombre andino en el desarrollo del país.

Los mestizos (o castas), llegaron a ser una cuarta parte de la población a inicios de la República. Tradicionalmente se considera así al hijo o hija de español con andina, pero, en este grupo social se considera también al mulato que es el hijo de español con negra; el cuarterón que era el descendiente de mulata y español y el zambo que era hijo de negro con mulata.

Los mestizos vivían especialmente en los pueblos y ciudades de la costa y, en un porcentaje menor en la sierra. Después de la independencia, ocuparon cargos políticos y públicos en las provincias, como era el caso de 
REFLEXIÓN SOBRE LA SITUACIÓN POLÍTICA, TERRITORIAL

Y SOCIAL DEL PERÚ DESPUÉS DE LA INDEPENDENCIA

los cabildos o recaudadores de tributos. Caso contrario, vivían de actividades del campo, especialmente de trabajar en chacras dejadas por los españoles.

A inicios de la República, habían en el territorio peruano, unos 40 mil esclavos negros, aunque su exportación fue a partir de la necesidad de llenar el vacío dejado por los indígenas en la mita minera colonial, en las primeras décadas del siglo XIX los encontramos en las haciendas costeras (azúcar, algodón, arroz entre otros productos).

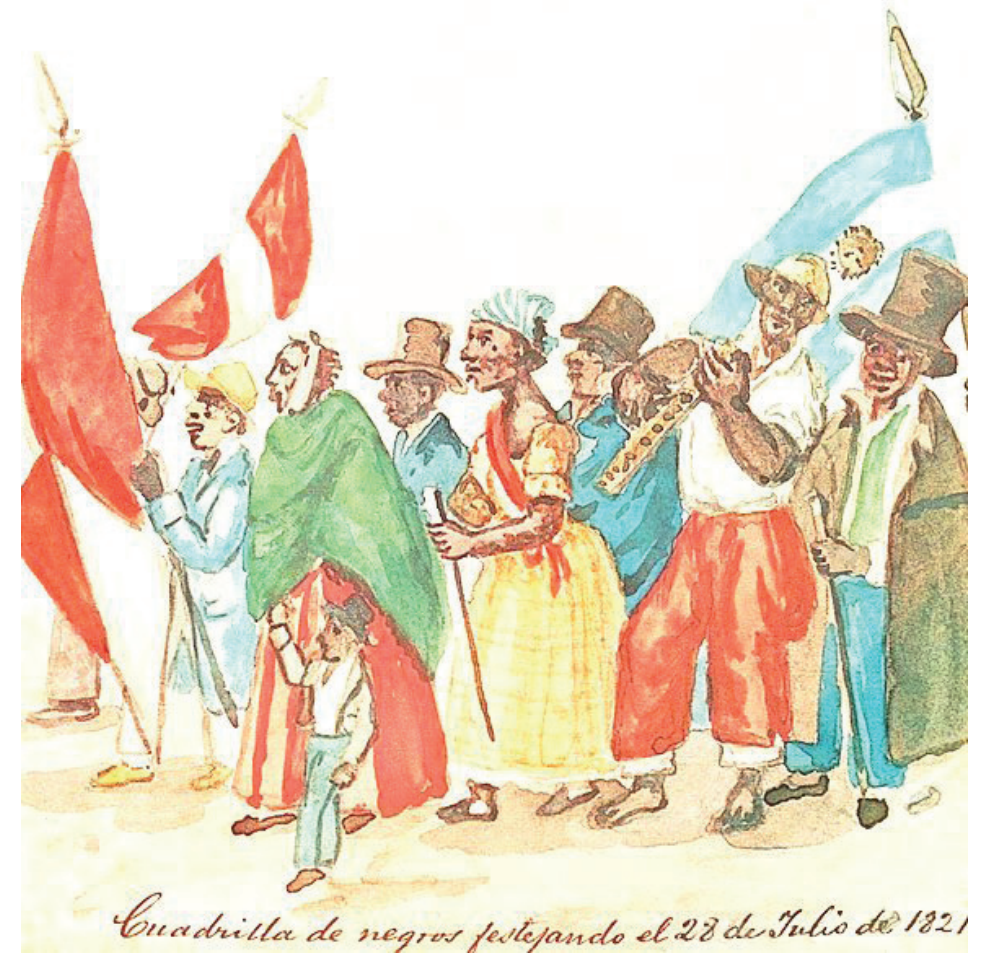

Figura 5. Negros festejando en el 28 de julio de 1821. Obra de Pancho Fierro. Tomado de https://www.wikiwand.com/es/Pancho_Fierro. 
Al igual que los andinos, los esclavos negros, vivieron el incumplimiento de las promesas independentistas, pero a diferencia de estos, la libertad de los esclavos tuvo que esperar hasta 1854 cuando Ramón Castilla, mediante Decreto, los reconoce como ciudadanos. Solamente los nacidos después de la proclama (1821) eran libres (pardos libres). Hecho que trajo como consecuencia un conflicto interno dentro del propio grupo social.

La ciudad de Lima ostentaba la mayor cantidad de esclavos en las primeras décadas del siglo XIX. Con el pasar del tiempo, se hizo difícil importar esclavos al Perú. Aunque se ha señalado que en 1854 obtuvieron su libertad, en la práctica no se llevó a cabo al cien por ciento, pues pasaron a la condición de servidumbre. Alcanzaron inclusive a obtener tierras para su propio provecho, o también salían a trabajar a las calles para el beneficio económico de sus amos.

Desde el punto de vista racial, la transición de Virreinato a República sigue igual, los grupos sociales "pasan" la brecha independentista y siguen viviendo con sus propias características y desigualdades sociales. Aunque, entre todos ellos, el menos favorecido es el andino, como se ha explicado.

Este crisol de grupos sociales, seguirá ampliándose con la llegada de otros, como es el caso de los chinos coolíes (a partir de 1849), alemanes y austriacos (1854), árabes (1885), judíos (1848), entre otros. De esta manera, el Perú de los siglos XX y XXI es considerado mestizo. 
REFLEXIÓN SOBRE LA SITUACIÓN POLÍTICA, TERRITORIAL

Y SOCIAL DEL PERÚ DESPUÉS DE LA INDEPENDENCIA

\section{Conclusiones}

T os acontecimientos mundiales de mediados del siglo XVIII influyeron en los ideales políticos de reformar o independizar los territorios que conformaban las colonias españolas en Latinoamérica. En lo concerniente al Perú, la influencia francesa fue la determinante, a diferencia de Venezuela que esperó el apoyo de los Estados Unidos. Asimismo, la Francia revolucionaria aportó en el sistema doctrinal político que tomarían los sectores criollos en el Perú (conservadores y liberales), esto último queda reflejado con la presencia de los próceres y precursores.

No siguió la misma suerte los movimientos insurgentes provinciales, esto porque la mayoría de ellos, surgían de un momento a otro y eran organizados sin la experiencia castrense. Unido a ello, el enfrentamiento a un ejército profesional como era el realista, y de manera especial, con una propuesta política de defensa militar por parte del virrey Abascal que se dio entre 1806 hasta 1816, hizo que el Perú tuviera que esperar hasta la llegada de la Corriente libertadora del Sur, dirigida por el general don José de San Martín. Sin la presencia del virrey Abascal la historia hubiera sido diferente, dándose probablemente el foco independentista en el Perú, tesis que debería de estudiarse.

La presencia del caudillismo y la diversidad de propuestas políticas por parte de los criollos, trajo como consecuencia que en las primeras décadas de su vida republicana, el Perú sea un espacio físico e intelectual en donde se ponga cronológicamente en práctica los diversos tipos de gobierno. Buscándose, de esta manera, intereses muchas veces foráneos, que llevaron inclusive a pérdidas territoriales. Unido a ello el gran distanciamiento entre las clases sociales, distanciamiento que hasta el día de hoy perdura en la sociedad peruana. 
Énfasis especial en el tema de la educación. Desde su independencia, el Perú no ha podido superar la situación de analfabetismo en la sociedad. Si se reflexiona sobre los hechos positivos y negativos del siglo XIX y mediados del XX, muchos de los errores de nuestra historia republicana se deben a las decisiones tomadas por las clases políticas letradas, es decir, llevaban bajo su responsabilidad la vida política de una sociedad que en su gran mayoría era iletrada. Mayoría que no tenía supuestamente la capacidad de decidir políticamente, viéndose esto reflejado en la ausencia de participación en los comicios electorales, situación que cambiará a mediados del siglo XX.

\section{REFERENCIAS}

Aljovín, C. (2000). Caudillos y Constituciones: Perú 1821-1845. Lima, Perú: Instituto Riva-Agüero, Fondo de Cultura Económica.

Alvear, C. (2004). Historia Universal Contemporánea. México, D.F., México: Editorial Limusa.

Congreso de la República. (s.f.). Diario de los Debates: Constitución de 1823. Recuperado de http://www4.congreso.gob.pe/dgp/ constitucion/constituciones/Constitucion-1823.pdf

Contreras, C., \& Cueto, M. (2000). Historia del Perú contemporáneo. Lima, Perú: Instituto de Estudios Peruanos.

De Agostini (Ed.). (2000). Absolutismo e Ilustración. Historia Universal (T. 8). Madrid, España: Gráficas Estrella. 
Del Busto, J. (2004). Conquista y Virreinato. Enciclopedia Temática del Perú (T. II). Lima, Perú: Editora El Comercio.

Kapsoli, W. (1995). Nacionalismo Inca. Lima, Perú: Editorial Purej.

Manrique, N. (2004). Sociedad. Enciclopedia Temática del Perú (T. II). Lima: Editora El Comercio.

Mariátegui, J. C. (2005). 7 Ensayos de interpretación de la realidad peruana. Lima, Perú: Orbis Ventures.

Milla, C. (Ed.). (1995). Atlas Geográfico y Documental del Perú (T. IV). Lima, Perú: Editorial Milla Batres.

Radio Programas del Perú Noticias. (2016). ¿Influyó la independencia de Estados Unidos en Perú y Latinoamérica? Recuperado de https:// rpp.pe/mundo/estados-unidos/influyo-la-independencia-deestados-unidos-en-peru-y-latinoamerica-noticia-976351

Real Academia Española. (2005). Diccionario de la lengua española (T. XVI). Lima, Perú: Q.W. Editores.

Historia Universal. (2006). En La Enciclopedia del Estudiante (T. 2). Lima, Perú: Santillana, Quebecor World Perú S.A.

Vargas, H. (2010). Antología de traiciones: breviario de los tratados del Perú. Lima, Perú: Editorial Rocío.

Villarrubia Marcelo, F. (2018). El Estado peruano 1. Realidad Nacional. El Estado: sus componentes, sus fines, sus instrumentos y sus medios. Bienestar Seguridad General Integral. SlidePlayer. Recuperado de https://slideplayer.es/slide/11808560/ 
